\title{
SPECT/CT imaging in breast cancer - current status and challenges
}

\author{
Milan Simanek', Pavel Koranda ${ }^{b}$
}

\begin{abstract}
Background. The increasing incidence of breast cancer worldwide raises the importance of improving imaging techniques for disease stratification after early lesion detection. SPECT/CT imaging is now widely available but its diagnostic potential is not fully utilized for more specific purposes including breast cancer patient stratification.

Methods and Results. A Pubmed search for both original and review articles related to the value of SPECT/CT in breast cancer patients and comparison to other diagnostic methods. 62 articles were found using the key words SPECT/CT, Fusion Image and Breast Cancer. Development of a new generation of SPECT/CT systems and their introduction into practice has changed the old diagnostic algorithm. The increasing importance of SPECT/CT in the detection of bone metastases is confirmed. The diagnostic accuracy of new SPECT/CT instruments in the diagnostics of bone metastases is nearly comparable to PET/CT scans. SPECT/CT is more widely available and costs less than PET. It is able not only to identify a sentinel lymph nodes in atypical localizations but also to detect sentinel lymph nodes non visualized on previous planar scans. SPECT/CT offers precise anatomic localization of sentinel lymph nodes, thereby facilitating surgery. Knowledge of precise sentinel lymph node localization can also be applied in radiotherapy.

Conclusions. The role of hybrid SPECT/CT imaging in breast cancer patients is changing. It is a powerful modality for skeletal and nodal staging in breast cancer patients with important impact on therapy.
\end{abstract}

Key words: breast neoplasms, sentinel lymph node biopsy, neoplasm metastasis, multimodal imaging, SPECT/CT

Received: May 9, 2016; Accepted with revision: June 28, 2016; Available online: August 2, 2016

http://dx.doi.org/10.5507/bp.2016.036

aDepartment of Nuclear Medicine, Regional Hospital Pelhrimov, Czech Republic

${ }^{b}$ Department of Nuclear Medicine, Faculty of Medicine and Dentistry, Palacky University Olomouc, Czech Republic

Corresponding author: Pavel Koranda, e-mail:pavel.koranda@fnol.cz

\section{INTRODUCTION}

The increasing incidence of breast cancer worldwide raises the importance of improvements in imaging techniques for disease stratification after early lesion detection.

Bone scintigraphy has been used for skeletal staging and has high sensitivity. The poor specificity of planar bone scans, however, has induced the need for more accurate diagnostic procedures. Development of new generation of SPECT/CT systems and their introduction into practice has changed the old diagnostic algorithm. SPECT/CT offers advantages in the detection of sentinel lymph nodes in breast cancer over planar imaging using the preferred lymphoscintigraphic technique. SPECT/ $\mathrm{CT}$ is able not only to identify sentinel lymph nodes in atypical localizations but also to detect sentinel lymph nodes non-visualized on previous planar scans. SPECT/ CT offers precise anatomic localization of sentinel lymph nodes, thereby facilitating surgery. The knowledge of precise sentinel lymph node localization can also be applied in radiotherapy.

This review outlines the role of hybrid SPECT/CT imaging as a powerful modality for skeletal and nodal staging in breast cancer patients with an important impact on therapy.

\section{FUNDAMENTALS OF HYBRID SPECT/CT IMAGING}

SPECT/CT hybrid imaging system allows doublemodality diagnostics with direct imaging fusion of morphological and functional information. We focused on the hybrid device SPECT/CT only in this article, not on the software based offline (from two separate devices) image fusion. The hybrid SPECT/CT system is composed of a gamma camera and a separate CT device with a shared bed for patients. The CT which is used is simple and designed especially for a gross morphological orientation and attenuation, or it can be multidetector CT (ref. ${ }^{1}$ ). According to the characteristics of CT scans used, a low dose CT and a fully diagnostic CT can be distinguished.

Integration of SPECT and CT image data is performed through the process known as image fusion. This process is based on highly sophisticated software tools that enable accurate spatial (3D) image overlap of two or more imaging modalities. The used automatic software must be able to eliminate inaccuracies due to differences in the position of the patient (while breathing or moving). Complementing this is an algorithm for manual adjustment of automatically collected fusion. A detailed overview of the principles of the image fusion in medicine in years is provided by James and Dasarathy $2014\left(\right.$ ref. $\left.^{2}\right)$. 


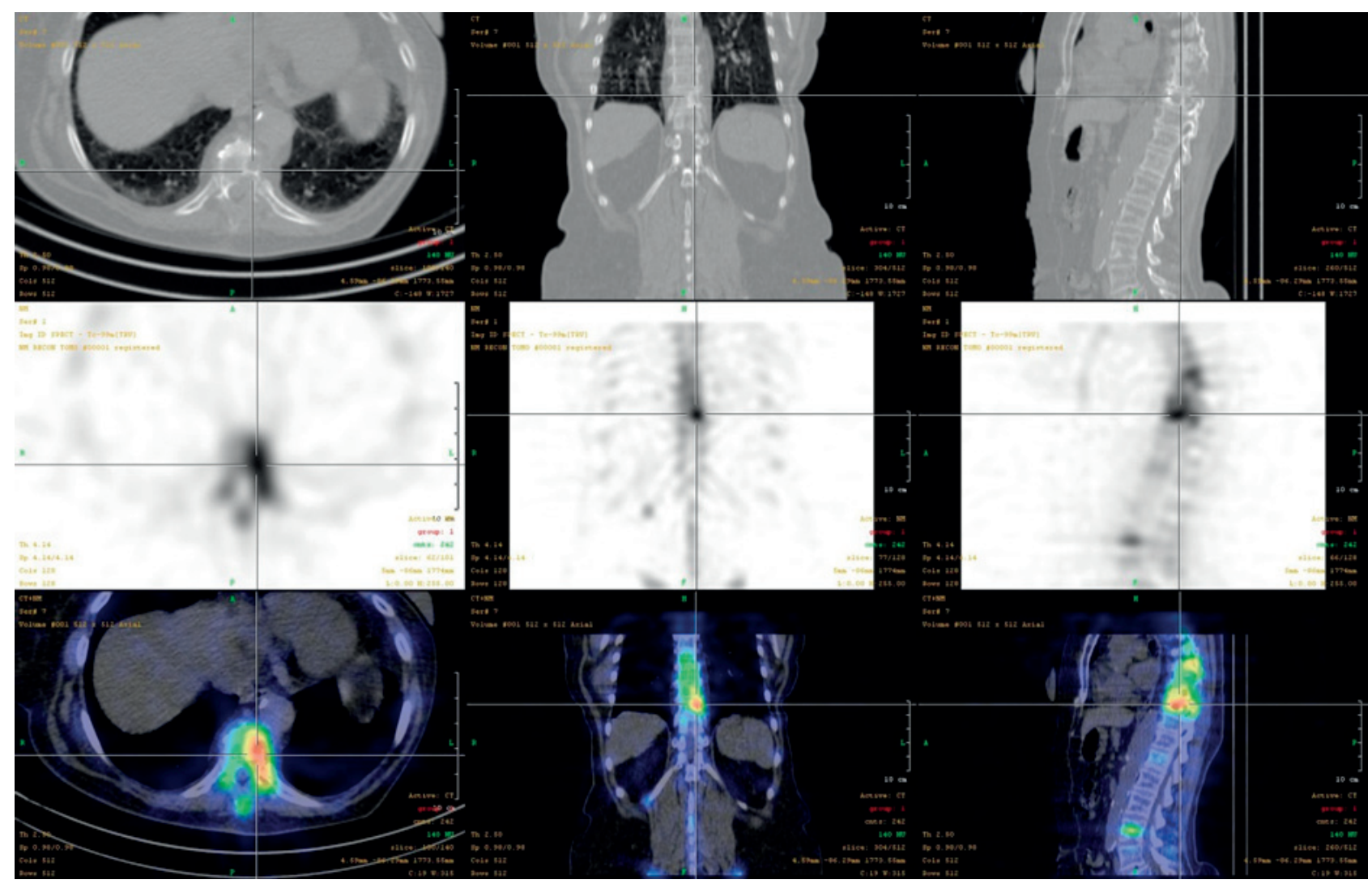

Fig. 1. Breast cancer with mixed bone metastases (top CTscan, centre SPECT scan, bottom fused SPECT/CT images).

Using the same international data format DICOM (Digital Imaging and Communications in Medicine $^{3}$ ) greatly facilitates the process of hybrid data fusion and makes possible processing, saving, printing or transferring the captured image information on different devices, for example via a PACS (Picture Archiving and Communicating System) (ref. $\left.{ }^{4}\right)$.

\section{ADVANTAGES OF THE FUSION IN HYBRID INSTRUMENTS}

Hybrid devices refine the algorithms used for the fusion. Input information on the spatial relationship of imaging data is provided by the hybrid device itself. A patient is lying on one examination bed; therefore, the data from both imaging modalities are consistent. Identical patient position during both image acquisitions allows an accurate co-registration of data. A 10\% improvement in diagnostic accuracy is achieved compared to the sequential examination on different devices with subsequent data fusion ${ }^{5}$.

The spatial co-registration of data obtained on $\mathrm{CT}$ and SPECT is important for the morphological localization of radiopharmaceuticals and even for attenuation correction. A poorly performed fusion may lead to false anatomical localization of scintigraphic findings. Even a good image fusion, however, has to count on possible errors - for example, with about $1 \mathrm{~mm}$ misalignment in the lumbar spine area ${ }^{6}$.

\section{THE BENEFITS OF SPECT/CT}

A number of methods used in nuclear medicine are presented as functional examinations describing the dynamic changes in the organism even prior reflection in the pathological morphological image. Additional techniques used in nuclear medicine are based on imaging the molecular characteristics of searched tissues that do not have a macroscopic correlate. However, a common feature of most methods used in nuclear medicine is the difficulty clinicians have in interpreting the image output due to a lack of a clear morphological context. The need to refine the diagnosis, anatomically localize lesions and present the results of scintigraphic studies to clinicians has become the goal of developing the SPECT/CT hybrid systems. An equally important task of the hybrid devices has been the correction of scintigraphic data distorted by irregular attenuation of the radiopharmaceutical photons in different tissues.

\section{ATTENUATION CORRECTION}

Attenuation of the radiation beam is a result of physical properties of tissues expressing the ability of tissue to reduce the number of photons in the radiation beam due to Compton scattering (for the energy of photopeak ${ }^{99 \mathrm{~m}} \mathrm{Tc} 141 \mathrm{keV}$, it is a dominant interaction of photons with the tissues in terms of attenuation) or absorption (photoelectric effect), and other phenomena at the atomic 


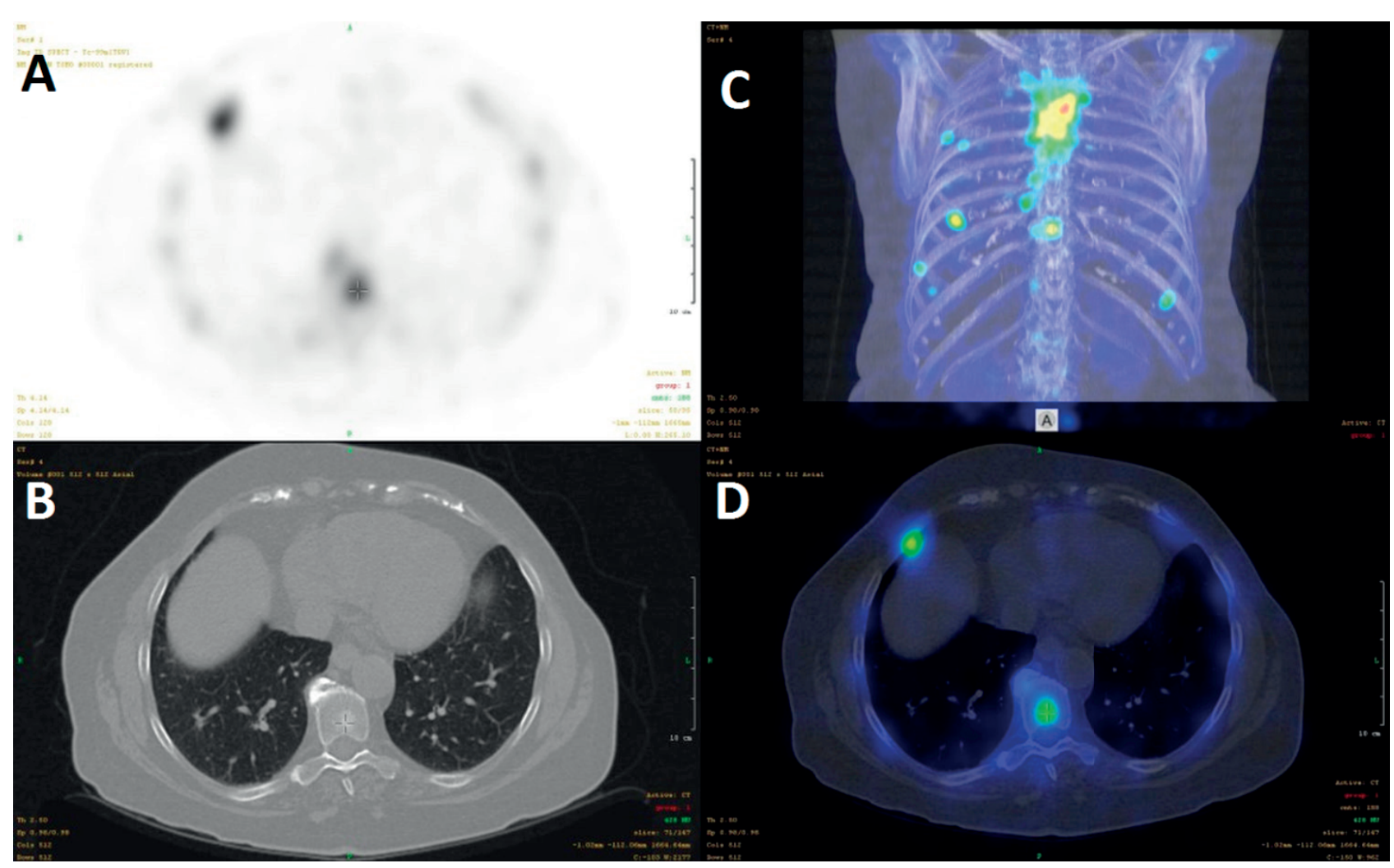

Fig. 2. Example of a poor fusion of metastasis in a rib during inspiration (false imaging of the focus outside the rib due to respiratory movement) and a precise fusion of focus in the vertebral body (A) SPECT image, (B) CT image, (C) 3D volume rendering fused SPECT/CT image, (D) fused SPECT/CT image.

level. The intensity of attenuation in any material can be described by a linear attenuation coefficient $(\mu)$ which is defined as the fraction of attenuated incident photons per unit thickness of a material. It primarily depends on the energy of photons and the density of tissue (effective atomic number of the $\mathrm{Z}_{\text {ef }}$ tissue).

Reducing the number of photons that reach a gamma camera detector after passing the tissues misrepresents the information in regard to amount of pharmaceutical in the organs under surrounding tissues. Coupled with partial volume effect this may lead to a deterioration in detecting smaller "hot" lesions (while doing a skeletal scintigraphy or receptor scintigraphy in breast cancer ${ }^{7}$ or in imaging the sentinel lymph node ${ }^{8}$ or an underestimation of "cold" regions (brain, heart ${ }^{9}$ ).

In the case of a homogeneous tissue structure (e.g. a brain in terms of $\mu$ value), the influence of attenuation can be approximately corrected using Chang's method applying the average attenuation coefficient ${ }^{10}$. If a tissue structure is more complex (e.g. a chest, where only a small attenuation occurs in the lung tissue and a considerably higher attenuation is in muscles, fat, and skeleton), a simple application of Chang's method is not enough. A three-dimensional map of the body organs with different attenuation coefficients must be created. Using of CT data is possible for this purpose (even low dose CT) (ref. ${ }^{9,11}$ ). Precise attenuation and scatter correction serves as a tool to achieve a good quality of scintigraphic images and enables a better calculation of target/non target ratio determining the intensity of radiopharmaceutical ${ }^{8}$ accumulation in the lesion. The nuclear-medical protocols can be shortened or the required doses of radiopharmaceuticals can be reduced ${ }^{12}$.

The use of CT data to correct attenuation of ${ }^{99 \mathrm{~m}} \mathrm{Tc}$ photons is complicated by the different characteristics of CT radiation attenuation. If typically a voltage of 120 $\mathrm{kV}$ on a CT X-ray tube is employed, the X-ray photon energy is lower than the $\gamma$-photon energy emitted by ${ }^{99 \mathrm{~m}} \mathrm{Tc}$ labeled radiopharmaceuticals $(141 \mathrm{keV})$. The theoretical use of two voltages for a CT attenuation scan is a possible improvement. Attenuation pitfalls may also be caused by metal implants in the body with a high $\mathrm{Z}_{\mathrm{ef}}$ as well as movements between the SPECT examination and the CT attenuation scan.

It is understood that the attenuation correction by a CT scan is very sensitive to a number of factors, and may not always lead to improved examination results. An inaccurate performance of an attenuation map for a distribution image of radiopharmaceuticals may cause "edge artifacts", i.e. the light and dark "circles" over the edges of these regions, caused by over- or undervaluing the attenuation. A proper assessment of SPECT images consists in a parallel evaluation of the corrected and uncorrected image so as to eliminate possible artifacts and monitor the quality of the examination itself. 


\section{ANATOMICAL LOCALIZATION}

The introduction of SPECT/CT implies an improved anatomical localization of pathological radiopharmaceutical accumulation (such as skeleton vs. soft tissue) (ref. ${ }^{13}$ ) even for the needs of other disciplines (surgery, radiotherapy) (ref. ${ }^{14}$ ). Compared to independent SPECT and CT examinations, the hybrid SPECT/CT examination provides an improved detection of lesions on SPECT and CT and confirmation of small or unusually localized lesions ${ }^{5}$.

Another benefit for the interpretation of scintigraphic findings is a better differentiation of the physiological and pathological distribution of radiopharmaceuticals based on:

- precise determination of anatomical structures (e.g. rib or costovertebral joint),

- localization in places where a tumorous process is unlikely (cartilage or joints)

- localization in places of a benign findings on CT (fractures, degenerative changes, typical benign changes on CT).

The result is an improved interpretation of scintigraphy ${ }^{1,15}$ and reduction in the number of additional examinations ${ }^{15}$.

\section{USING SPECT/CT HYBRID SYSTEMS FOR PATIENTS WITH BREAST CANCER}

The use of the hybrid imaging is possible at the initial diagnosis, diagnosis of disease complications, bone metastasis radiotherapy planning ${ }^{14}$, monitoring the response to treatment and treatment results, and finally, further monitoring of patients ${ }^{6}$. However, routine adaptation of hybrid examination is not routine, partly because of the large variety of systems used. Only $51 \%$ of workplaces which were equipped with the hybrid systems in 2010 used the SPECT/CT routinely ${ }^{16}$. In recent years, specialists have explored the added value of integrating SPECT gamma cameras with fully diagnostic CT scanner ${ }^{17}$.

For patients with breast cancer, the hybrid SPECT/CT imaging currently has the greatest effect for:

1. Detection of bone metastases and differential diagnosis of benign and extraskeletal lesions

2. Accurate mapping of lymphatic drainage of the breast before performing a radioguided sentinel lymph node biopsy.

\section{Detection of bone metastases}

A series of studies evaluating the importance of bone SPECT/CT in patients with breast cancer has been published over the last decade. However, recent articles based on the usage of the most advanced devices have the highest value for the assessment of the current importance of SPECT/CT in breast cancer patients.

Earlier studies have confirmed a high incidence of metastatic bone disease in advanced breast cancer ${ }^{18}$. The scintigraphic detection of bone metastases of often osteoblastic or a mixture of osteolytic and osteoblastic type is commonly used. Purely lytic lesions can go undetected, however, only while performing a simple whole-body planar scintigraphy. A further examination is also required for equivocal lesions which are often of a degenerative or posttraumatic origin ${ }^{19}$.

The advantage of the SPECT/CT hybrid systems in detecting bone metastases has been recently described by many authors. Azad 2015 (ref. ${ }^{20}$ ) ascertained the reduction of false negative and false positive findings using SPECT/CT versus a planar skeletal scintigraphy; Withofs ${ }^{21}$ reported essentially the same. Palmedo ${ }^{15}$ demonstrated a significantly improved specificity and positive predictive value of SPECT/CT in comparison to planar bone scintigraphy and, in addition, found further improvement in sensitivity particularly in patients with breast cancer. This defines the extent of metastases better and reduces the need for additional diagnostic procedures. According to this author, sensitivity $96 \%$, specificity $95 \%$, positive $99 \%$ and negative predictive values $87 \%$ are achieved for the detection of bone metastases using bone SPECT/CT in women with breast cancer. The hybrid SPECT/CT imaging contributed to downstaging of the disease in $33.8 \%$; to upstaging in $2 \%$ of patients. Only $2 \%$ of patients had to be examined further.

Using the hybrid systems with a good SPECT and CT image co-registration reduces the number of indeterminate lesions on scintigraphy itself by a characteristic anatomical localization of lesions to the pedicles, intervertebral or costovertebral joints, the edges or centres of vertebral bodies or vertebral arches ${ }^{22}$. The CT characteristics of lesions based on the presence of osteolysis or sclerosis is another important piece of information.

Reduction in the number of indeterminate lesions on skeletal scintigraphy has been found by other authors in particular in patients with lytic lesions ${ }^{23}$. SPECT/CT is able to correctly interpret $96 \%$ of lesions in the vertebrae imaged using a bone scintigraphy. This resulted in a change in treatment of patients in $61 \%$ compared to a planar scintigraphy and in $18 \%$ compared to SPECT (ref. ${ }^{24}$ ). Concerning the examination of patients with back pain, there was a threefold improvement of the characteristics of indeterminate lesions by SPECT/CT versus SPECT $\left(\right.$ ref. $\left.^{25}\right)$. For solitary lesions in the spine, SPECT/CT increases the sensitivity of bone metastases detection from $6 \%$ using planar scan, to $79 \%\left(\right.$ ref. $^{26}$ ).

Further improvement is documented when determining the nature of the lesions in the skull using SPECT/ CT (in 100\%) (ref. ${ }^{27}$ ). For the differential diagnosis of osteonecrosis of the jaw using bisphosphonate therapy or metastasis to the jaw, the use of SPECT/CT is needed ${ }^{28}$.

Unlike the above cited authors, Haraldsen (ref. ${ }^{29}$ ) found no substantial improvement of the SPECT/low dose CT diagnosis of bone metastases in breast cancer compared to the whole-body planar scan but the sample size was small. Haraldsen reported sensitivity of SPECT/ low dose CT $79 \%$ only, in contrast with sensitivity of whole-body planar scan $87 \%$. Specificity was estimated at only $63 \%$ in both methods. Low-dose CT was performed in this study with the parameters of $120 \mathrm{kV}, 60 \mathrm{mAs} /$ slice, $600 \mathrm{~mm}$ FOV, a matrix of $512 \times 512,5 \mathrm{~mm}$ slices, a pitch factor of 0.875 , collimation $8 \times 3$, for the low dose CT. 
An improvement in the specificity of the hybrid system (83\%) was found when SPECT/full diagnostic CT with an administration of intravenous contrast medium was performed (with the parameters of $120 \mathrm{kV}, 200 \mathrm{mAs} / \mathrm{slice}$, $300 \mathrm{~mm}$ FOV, a matrix of $512 \times 512,2 \mathrm{~mm}$ slices, a pitch factor 0.938 , collimation $16 \times 1.5)$.

\section{Differentiation of benign lesions}

The precise anatomic localization of lesions improves the diagnostics of the benign nature of radiopharmaceutical deposits, e.g. at the spine in the region of facet joints in up to $100 \%$ of $\operatorname{cases}^{30}$. SPECT/CT is also able to determine the benign character of different bone lesions: spine hemagiomas $^{31}$, Schmorl's nodes ${ }^{32}$, pelvic fractures ${ }^{33}$. In case of isolated lesions of the spine, Iqbal ${ }^{26}$ established a benign diagnosis in $80 \%$ of patients using SPECT/CT these were cases of osteomyelitis, post-traumatic changes, spondylolysis, spondylolisthesis, and osteoarthritis of facet joints.

\section{Comparison with hybrid systems using PET}

PET technology in combination with standard fully diagnostic CT or MR imaging and the use of either a universal tumour-specific radiopharmaceutical ${ }^{18} \mathrm{~F}-\mathrm{FDG}$ or a specific radiopharmaceutical for skeleton $\mathrm{Na}^{18} \mathrm{~F}$ can provide a more accurate diagnosis of bone metastases.

Cook 2016 (ref. ${ }^{34}$ ), Jambor 2016 (ref. ${ }^{35}$ ), Caglar 2016 $\left(\right.$ ref. ${ }^{36}$ ), and others reported better sensitivity and specificity of PET/CT compared to SPECT/CT (sensitivity increases from $85-89.5 \%$ obtained with the SPECT/CT to $93 \%$ for $\mathrm{Na}^{18} \mathrm{~F}$ up to $97.6 \%$ for ${ }^{18} \mathrm{~F}-\mathrm{FDG}$ ). Even more advantageous seems to be the ${ }^{18} \mathrm{~F}$-FDG PET/standard fully diagnostic CT compared to the planar skeletal scintigraphy (sensitivity 96\% vs. 76\%) (ref. ${ }^{37}$ ). Some authors observed poorer detection of metastases particularly in the pelvis, spine and sacrum with the planar scintigraphy ${ }^{38}$. The greatest benefit of SPECT/CT is reported, however, for these localizations ${ }^{24}$. The authors drew attention to the lower availability and higher cost of PET/CT versus SPECT/CT for a routine check-up ${ }^{34}$. Jambor ${ }^{35}$ and Hahn ${ }^{37}$ reported about a slight increase in the proportion of indeterminate lesions on the PET/CT scans for the detection of bone metastases in high risk breast cancer patients.

For this reason, some authors subsequently recommended the deployment of PET/CT for uncertain findings on SPECT/CT skeleton, even in the form of a $\mathrm{Na}^{18} \mathrm{~F}^{-18} \mathrm{FDG}$ PET/CT "cocktail” (ref. ${ }^{39}$ ). The combination of multiple methods is optional and possible ${ }^{40}$ but provides only a slight improvement in the diagnosis of bone metastases. Green ${ }^{41}$ drew attention to the advisability of further research in the use of new SPECT/CT systems in conventional skeletal scintigraphy, even at a high diagnostic value of PET/CT.

Currently, interest and experience in using whole-body morphologic MRI augmented by diffusion weighted imaging for both staging and response assessment in the skeleton have been increasing. However, data on comparisons of these methods with PET methods to determine the best technique for current clinical practice or for clinical trials are insufficient. There are early data supporting the use
18F-FDG PET/MRI (include diffusion weighted imaging) to assess malignant disease in the skeleton, with the possibility of taking advantage of the synergies offered by combining morphologic, physiologic and metabolic imaging ${ }^{34,35}$.

\section{Targeting radiotherapy and assessment of response to therapy}

Sergieva ${ }^{14}$ considers SPECT/CT beneficial to improving the targeting for the radiotherapy of metastatic lesions of breast cancer within the parameters of GTV - Gross Tumour Volume and CTV - Clinical Target Volume. She also describes the usefulness of SPECT/CT evaluation of bone lesion consolidation and accumulation of calcium after treatment.

Usability of scintigraphy itself is refuted by $\mathrm{Cook}^{34}$ who stated that only $52 \%$ of patients responded to therapy even with an improved finding on scintigraphy after 6-8 months. At the same time, $62 \%$ of patients who do not respond well to therapy have noticeable deterioration on the skeletal scintigraphy. Additionally, Cook refers to a well-known flare phenomenon on the skeletal scintigraphy after the administration of chemotherapy or endocrine therapy for breast cancer. This led to an undermining of the effect of therapy on bone lesions 3-6 months after its implementation. The author believes, therefore, that using SPECT/CT evaluation of the effect of therapy does not improve the results and for this reason, he suggests the use of ${ }^{18} \mathrm{~F}-\mathrm{FDG} \mathrm{PET} / \mathrm{CT}$, with regard to the presence of flare phenomenon even in $\mathrm{Na}^{18} \mathrm{~F}$ PET/CT.

\section{Extraskeletal lesions}

SPECT/CT also localizes extraskeletal lesions during skeletal scintigraphy, including extraskeletal metastases whether accumulating or not accumulating ${ }^{42}$ a bone radiopharmaceutical ${ }^{13}$. The latter outlines a range of clinical cases of breast cancer. The extraskeletal uptake of phosphonates occurs in some malignant pleural or pericardial effusions, liver or lymph metastases or necrotic tissue non-malignant calcifications. Other example are metabolic diseases leading to hypercalcaemia and tissue calcification and the extraskeletal uptake of radiopharmaceuticals: renal failure with hyperparathyroidism, amyloidosis, urogenital system disorders (nephritis, lithiasis) or post-traumatic lesions with muscle damage.

Performing the SPECT/CT of a chest for a better characterization of vertebral deposits of radiopharmaceuticals or for a staging of advanced breast cancer may even lead to the incidental finding of pulmonary metastases ${ }^{43}$.

\section{Accurate mapping of lymphatic drainage of the breast}

Radioguided sentinel lymph node biopsy (SLNB) has become an important tool for accurate $\mathrm{N}$ staging of breast cancer, particularly in the early T1 and T2 stages (TNM system) with clinically negative axillary lymph nodes. Patients with a preoperative metastatic axillary ultrasonographic findings are not indicated for SLNB. SLNB in all different versions clearly provides valuable staging information with less morbidity than a standard axillary lymph node dissection (ALND). It minimizes the risk of lymph- 


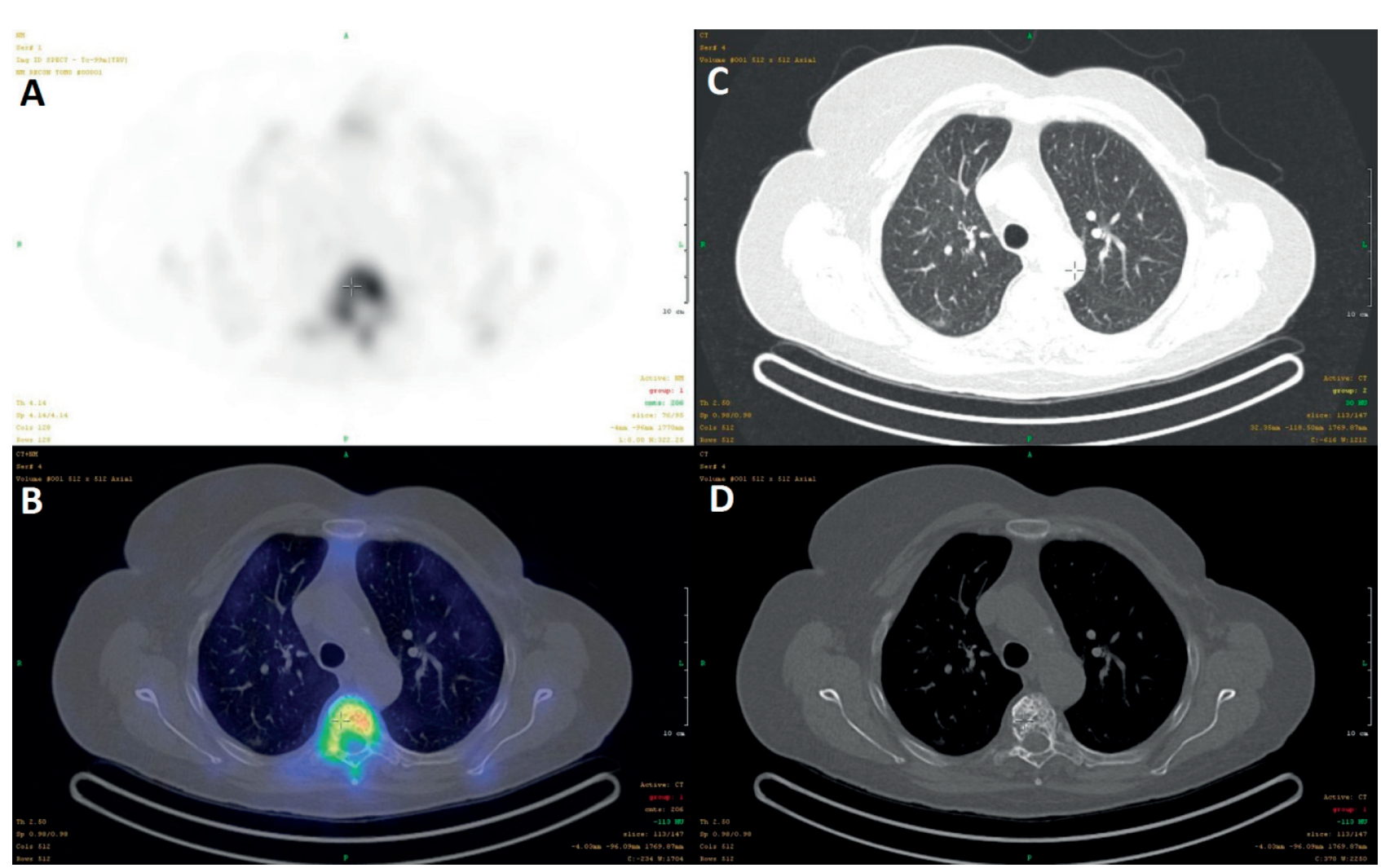

Fig. 3. Bone and lung window in evaluating the fused image with a mixture of metastases in the spine. (A) SPECT image, (B) fused SPECT/CT image, (C) CT image, lung window, (D) CT image bone window.

edema. The researchers randomly assigned participants to receive SLNB alone or SLNB plus ALND and they found no differences in overall survival and disease-free survival between the two groups of women. (NSABP-B32, ACOSOG-Z0011, Sentinella-GIVOM, Milan).

Lymphographic detection of the sentinel lymph nodes enables a targeted detailed histopathologic examination of directly draining lymph nodes extirpated by a surgeon during a radiation navigated surgery. The accurate preoperative lymphoscintigraphic determination of the number, localization and activities of the draining lymph nodes makes the surgery faster and more accurate ${ }^{44}$.

The recent EANM guideline points out that despite the widespread use of SLNB for early-stage breast cancer, there is a significant variation in performance characteristics reported for this procedure. Differences in lymphatic mapping techniques are important factors contributing to variations in the success rate of identification of SLN. One source of this irregularity is variable usage of SPECT/ CT. According to the guidelines, current indications for SPECT/CT include non-visualization of SLN on conventional planar imaging, patient obesity, and the presence of extraaxillary SLNs, or otherwise unusual difficult-tocharacterize drainage. The guideline was published in 2013, but the discussion about these recommendations continues. Some centers do not use SPECT/CT at all, some use it for all patients and others use it in accordance with EANM guidelines.

The following paragraphs comment on the different results of SPECT/CT in breast cancer patients.

\section{SPECT/CT in patients with non-visualization of SLN on planar imaging}

EANM guideline recommends SPECT/CT in case of non-visualisation of SLN on planar lymphoscintigraphy. In a recent IAEA multicentre study ${ }^{48}$ SPECT/CT increased the visualisation rate from $87.6 \%$ to $91.3 \%$. However, an alternative way for increasing SLN visualisation is tracer reinjection and Pouw ${ }^{49}$ compared both procedures in patients with SLN non-visualisation after intra-tumoural tracer administration. The SPECT/CT visualisation rate was $23 \%$. In patients receiving reinjection after persistent SPECT/CT non-visualisation the SLNvisualisation rate reached $62 \%$. It should be borne in mind that the main objective of SLN evaluation is not an imaging, but successful intraoperative detection. The surgical identification rate in the visualisation group on SPECT/ CT without reinjection was $77 \%$, the percentage was relatively low because the SLNs contained small amounts of radioactivity. The value of the same parameter in the group of patients after reinjection was $94 \%$. The authors of this study later readjusted their protocol in favour of reinjection. They reserve SPECT/CT imaging for patients with persistent absence of drainage after reinjection. This recent study could play an important role when EANM guidelines are updated.

\section{SPECT/CT increases the number of displayed lymph nodes}

Serrano-Vicente ${ }^{45}$ reported improvement in the detection of sentinel lymph nodes (SLN) before the SLNB 


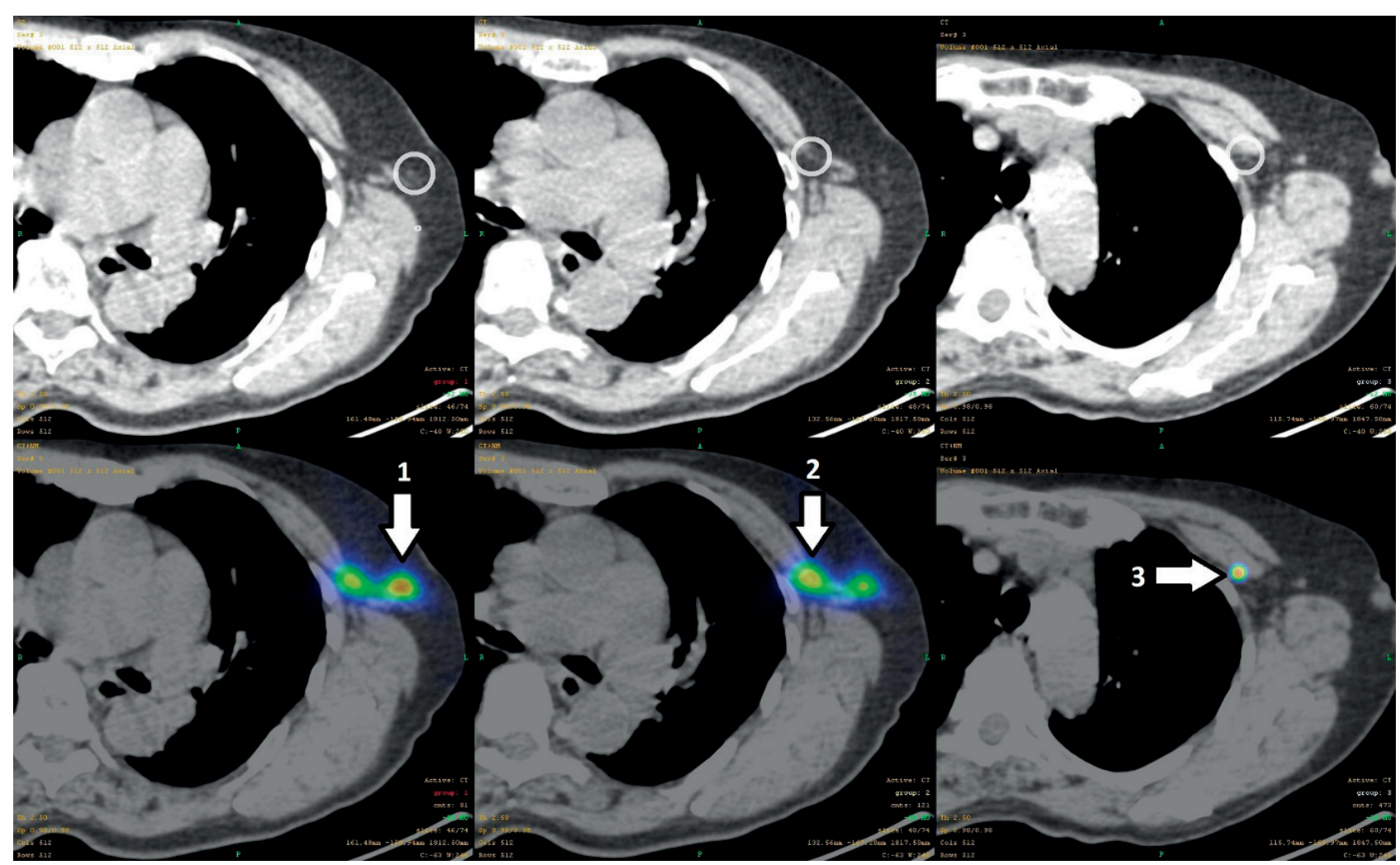

Fig. 4. Imaging of lymph node chain in the direction of lymph flow right to left - from the surface (1) up to deeply subpectorally localized lymph nodes (3).(CT images top, fused SPECT/CT images bottom).

itself, in relation to the number of lymph nodes and their localization by SPECT/CT. He found an $18 \%$ increase in the number of displayed lymph nodes accumulating lymphoscintigraphic radiopharmaceutical after its peritumoral application. Similarly $10 \%$ increase in the number of displayed lymph nodes by SPECT/CT was reported by Lerman ${ }^{46}$.

\section{SPECT/CT improve the localization of displayed lymph nodes}

Wagner ${ }^{47}$ described the benefits of using SPECT/CT in a precise anatomical localization of lymph nodes with a relationship to surrounding anatomical structures. $\mathrm{He}$ confirmed the identification of additional lymph nodes that were not visible on a planar scan and highlighted the reduction of potential artefacts (a skin contamination) or technical difficulties after application of the radiopharmaceutical. According to a prospective multicentre study organized by IAEA ( ref. $^{48}$ ), the SPECT/CT provides a more accurate localization of the SLN previously displayed on planar scans after peritumoral or subareolar application of radiopharmaceuticals or a combination. Part of the SLN displayed on planar scans in the axilla was localized using SPECT/CT much more precisely in the interpectoral or intramammary region. A possible metastatic involvement of SLN, which only appeared on SPECT/CT, was also highlighted in this study.

Ure $^{44}$ evaluated lymphatic drainage to the axillary lymph nodes and described the alternative directions of drainage proven through the accurate SPECT/CT localization.

\section{Multifocal and multicentric tumours with multiple applications of the radiopharmaceutical}

The precise determination of anatomical localization of individual drainages using SPECT/CT is needed for multifocal and multicentric tumours ${ }^{50}$. Brower found that additional lymphatic drainages were depicted after injection into the second or the third tumour(s) in $64 \%$ of patients. Sentinel lymph nodes were localized in a different lymphatic basin than sentinel lymph nodes identified after the first injection in $56 \%$ of patients. Some of these sentinel nodes were only seen on SPECT/CT, and not visualized on planar images.

\section{Evaluation of CT image of SLN}

SPECT/CT can alert physicians to the possibility of the metastatic involvement of lymph nodes in the axilla depending on their shape and size $\mathrm{e}^{51,52}$. The CT-based criteria for SLN metastases were as follows: lymph node size of $1 \mathrm{~cm}$ or more in smallest diameter, long-to-short-axis ratio 2 or lower, irregular margin ${ }^{51}$. $\mathrm{Na}^{52}$ found a greater ratio of long-to-short-axis of uninvolved lymph node versus metastatic lymph node (2.38 vs. 2.04).The metastatic lymph nodes were more rounded or with an eccentric edge, non-metastatic were more $\mathrm{C}$ shaped. Unfortunately, the accuracy of CT scanning in identifying metastatic disease in lymph nodes is limited. In the case of CT suspicion of metastases in lymph nodes, further examination (e.g. ultrasound, biopsy or PET) is desirable. 


\section{Extraaxillar lymph nodes}

SPECT/CT provides an advantage in the better detection of extraaxillar SLN. Serrano - Vicent ${ }^{45}$ compared to planar lymphoscintigraphy and SPECT/CT. They found that $10 \%$ (7 lymph nodes) of SLN imaged using scintigraphy in the internal mammary chain (IMC) region are mediastinal lymph nodes, another 8 mediastinal lymph nodes were displayed only on SPECT/CT. A more accurate localization of IMC lymph nodes on SPECT/CT is suitable for possible surgical intervention ${ }^{53}$.

\section{Detection of complications of lymph node dissection - chyloma}

Thang $^{54}$ reported the suitability of the SPECT/CT technique for detecting a chyloma after previous axillary lymph node dissection.

\section{SPECT/CT and individualisation of tangential radiation therapy}

However, Wadasaki ${ }^{55}$ has pointed out the possibility of using data from the SPECT/CT localization of SLN to plan lymph node irradiation. Modification of the individual treatment fields according to the SLN location seemed to be necessary to ensure coverage of the SLNs in whole-breast irradiation. Wadasaki used the SPECT/ CT to determine the field edge of a postoperative tangential irradiation of the breast and the area of the SLN location as well. He found a mean dose to SLN 95.8\% of the prescribed dose in this case. Reznik ${ }^{56}$ found, on the contrary, the mean axillary dose $66 \%$ of the prescribed dose only without the individual modification of the irradiation field.

\section{Radiation burden and the need for justification for performing SPECT/CT}

Using the SPECT/CT technique in SLNB leads to a slight increase in an effective dose (SPECT effective dose is about $1 \mathrm{mSv}$ for $50 \mathrm{MBq}{ }^{99 \mathrm{~m}} \mathrm{Tc}$ - nanocolloid and the total effective dose SPECT/CT is up to $3 \mathrm{mSv}$ according to $\mathrm{Law}^{57}$ ), thus the use of a hybrid system should always be justified by the expected benefit ${ }^{58-62}$. A low-dose CT scan can help keep the radiation dose to a minimum.

For bone scan the SPECT effective dose is about 4.2 $\mathrm{mSv}$ for $740 \mathrm{MBq} 99 \mathrm{mTc}-\mathrm{MDP}$. There is a wide range of used characteristics of CT scans in the hybrid SPECT/ CT device. There is a distinction between a low dose CT and a fully diagnostic CT. Avramova-Cholakova ${ }^{63}$ reported effective doses for four SPECT-CT systems: two Symbia 2 $\mathrm{T}$ (Siemens Medical Solution) equipped with a 2-detector row CT, Symbia T16 (Siemens Medical Solution) with a 16-detector row CT and one Discovery NM/CT670 (GE Healthcare) with 16-detector row CT. For bone imaging and different anatomical regions, CT doses in Symbia 2 $\mathrm{T}$ and Symbia T16 are similar (1.2 and $1.8 \mathrm{mSv}$, respectively) and much higher, $7.2 \mathrm{mSv}$, for the Discovery NM/ CT670.

The effective dose can be lower in both cases, a low dose CT and a fully diagnostic CT, using iterative reconstructions in comparison to filtered back projection. In the prospective study of Sibille ${ }^{64}$, enrolled patients underwent bone SPECT/CT with one SPECT acquisition followed by two randomized CT acquisitions: FBP CT and iterative reconstructions CT. CT acquisitions were performed using helical rotation, $120 \mathrm{kV}$; automatic tube current modulation, max range $10-350 \mathrm{~mA}$. The mean CTDIvol, DLP and effective dose for iterative reconstructions $\left(3.0 \pm 2.0 \mathrm{mGy} ; 148 \pm 85 \mathrm{mGy}^{*} \mathrm{~cm}\right.$ and $\left.2.2 \pm 1.3 \mathrm{mSv}\right)$ were significantly lower than for filtered back projection (8.5 $\pm 3.7 \mathrm{mGy} ; 365 \pm 160 \mathrm{mGy} * \mathrm{~cm}$ and $5.5 \pm 2.4 \mathrm{mSv}$ ).

Qualitative image analysis showed no qualitative differences between filtered back projection and iterative reconstructions of CT images for different readers.

\section{CONCLUSION}

The rising incidence of breast cancer worldwide increases the importance of improving imaging techniques for disease stratification after early lesion detection. The SPECT/CT hybrid systems change the examination algorithms, including the case of patients with breast cancer.

Bone scintigraphy has been used for skeletal staging and has a high sensitivity. Poor specificity of planar bone scan, however, has induced the need for more accurate diagnostic procedures. The diagnostic accuracy of new SPECT/CT devices in the diagnostics of bone metastases is, according to present studies nearly comparable to ${ }^{18} \mathrm{~F}-\mathrm{PET} / \mathrm{CT}$ or ${ }^{18} \mathrm{~F}-\mathrm{FDG} \mathrm{PET} / \mathrm{CT}$ scans. SPECT/CT is more widely available with costs less than PET techniques, which are not able to fully substitute this method in everyday practice. In contrast, evaluation of the effect of therapy using ${ }^{18} \mathrm{~F}-\mathrm{FDG} \mathrm{PET} / \mathrm{CT}$ is more accurate than bone scanning.

SPECT/CT offers also advantages in the detection of the sentinel lymph nodes (SLNs) in breast cancer. The main current indications are: localization of extra-axillary SLNs (precise anatomic localization facilitating surgery of SLNs outside the axillary Level I) and non-visualization of SLNs on planar images or difficulties in interpreting drainage on planar scans. Recent data suggest further appropriate applications - differentiation of internal mammary and mediastinal SLNs and individualization of treatment in radiotherapy. Current data reevaluate the role of SPECT/CT in patients with non-visualization of SLNs - reinjection of radiopharmaceuticals exhibits higher efficiency in SLNs detection than SPECT/CT only.

The role of hybrid SPECT/CT imaging in breast cancer patients is changing. It is a powerful modality for skeletal and nodal staging in breast cancer patients with an important impact on therapy.

Author contribution: Both authors contributed equally to preparing the manuscript.

Conflict of interest statement: The authors state that there are no conflicts of interest regarding the publication of this article. 


\section{REFERENCES}

1. Leitha T, Staudenherz A. Hybrid PET/CT and SPECT/CT Imaging. In Saba L, editor. Computed Tomography - Clinical Applications. InTech 2012; 269-94.

2. James AP, Dasarathy BV. Medical Image Fusion: A survey of the state of the art. Information Fusion 2014.

3. Mildenberger P, Eichelberg M, Martin E. Introduction to the DICOM standard. Eur Radiol 2002;12(4):920-7.

4. Branstetter BF 4th. Basics of imaging informatics. Part 1. Radiology 2007;243(3):656-67.

5. Delbeke D, Schöder H, Martin WH, et al. Hybrid imaging (SPECT/ CT and PET/CT): improving therapeutic decisions. Semin Nucl Med 2009;39(5):308-40.

6. Clinical Applications of SPECT/CT: New Hybrid Nuclear Medicine Imaging Systém. IAEA-TECDOC-1597, Vienna 2008

7. Meng Q, Li Z. Molecular imaging probes for diagnosis and therapy evaluation of breast cancer. Int J Biomed Imaging 2013; Article ID 230487.

8. Yoneyama H, Tsushima $\mathrm{H}$, Onoguchi M, Konishi T, Nakajima $\mathrm{K}$ Matsuo S, Kayano D,Wakabayashi H, Inaki A, Kinuya S. Optimization of attenuation and scatter corrections in sentinel lymph node scintigraphy using SPECT/CT systems. Ann Nucl Med 2015;29(3):248-55

9. Lee TC, Alessio AM, Miyaoka RM, Kinahan PE. Morphology supporting function: attenuation correction for SPECT/CT, PET/CT, and PET/ MR imaging. Q J Nucl Med Mol Imaging 2016;60(1):25-39.

10. Gillen R, Firbank MJ, Lloyd J, O'Brien JT. CT-based attenuation and scatter correction compared with uniform attenuation correction in brain perfusion SPECT imaging for dementia. Phys Med Bio 2015;60(17):6775-87.

11. Patton JA, Turkington TG. SPECT/CT physical principles and attenuation correction. J Nucl Med Technol 2008;36(1):1-10.

12. Palyo R, Sinusas A, Liu YH. High-Sensitivity and High-Resolution SPECT/CT Systems Provide Substantial Dose Reduction without Compromising Quantitative Precision for Assessment of Myocardial Perfusion or Function. J Nucl Med 2016;57(6):893-9. [Epub ahead of print] doi: 10.2967/jnumed.115.164632.

13. Soundararajan R, Naswa N, Sharma P, Karunanithi S, Nazar AH, Das $\mathrm{KJ}$, Bal C, Malhotra A, Kumar R. SPECT-CT for characterization of ex traosseous uptake of 99mTc-methylene diphosphonate on bone scintigraphy. Diagn Interv Radiol 2013;19(5):405-10.

14. Sergieva S, Mihaylova I, Alexandrova E, Dimcheva M, Mansi L. SPECTCT in Radiotherapy Planning, with Main Reference to Patients with Breast Cancer.Curr Radiopharm 2015;8(1):9-18.

15. Palmedo $H$, Marx C, Ebert A, Kreft B, Ko $Y$, Türler A, Vorreuther $R$ Göhring U, Schild HH, Gerhardt T, Pöge U, Ezziddin S, Biersack HJ, Ahmadzadehfar H. Whole-body SPECT/CT for bone scintigraphy: diagnostic value and effect on patient management in oncological patients. Eur J Nucl Med Mol Imaging 2014;41(1):59-67.

16. Wieder H, Freudenberg LS, Czernin J, et al. Variations of clinical SPECT/CT operations: an international survey. Nuklearmedizin 2012;51(4):154-60.

17. Ahmadzadehfar $\mathrm{H}$, Biersack $\mathrm{H}$, editoři. Clinical Applications of SPECTCT. Springer 2014; 201-16

18. Hagberg KW(1), Taylor A, Hernandez RK, Jick S. Incidence of bone metastases in breast cancer patients in the United Kingdom: results of a multi-database linkage study using the general practice research database. Cancer Epidemiol 2013;37(3):240-6.

19. Houssami N, Costelloe CM. Imaging bone metastases in breast cancer: evidence on comparative test accuracy. Ann Onco 2012;23(4):834-43.

20. Azad GK, Taylor B, Rubello D, Colletti PM, Goh V, Cook GJ. Molecular and Functional Imaging of Bone Metastases in Breast and Prostate Cancers: An Overview. Clin Nucl Med 2015;41(1):e44-50.

21. Withofs N(1), Collignon J, Hustinx R. Imaging bone metastases in breast cancer. Rev Med Liege 2011;66(5-6):291-8.

22. Ghosh P. The role of SPECT/CT in skeletal malignancies. Semin Musculoskelet Radiol 2014;18(2):175-93.

23. Sharma P, Singh H, Kumar R, Bal C, Thulkar S, Seenu V, Malhotra A. Bone scintigraphy in breast cancer: added value of hybrid SPECTCT and its impact on patient management. Nucl Med Commun 2012;33(2):139-47.

24. Sharma P, Dhull VS, Reddy RM, Bal C, Thulkar S, Malhotra A, Kumar R. Hybrid SPECT-CT for characterizing isolated vertebral lesions ob- served by bone scintigraphy: comparison with planar scintigraphy, SPECT, and CT. Diagn Interv Radiol 2013;19(1):33-40.

25. Zhang Y, Shi H, Gu Y, Xiu Y, Li B, Zhu W, Chen S, Yu H. Differential diagnostic value of single-photon emission computed tomography/spiral computed tomography with Tc-99m-methylene diphosphonate in patients with spinal lesions. Nucl Med Commun 2011;32(12):1194200.

26. Iqbal B, Currie GM, Wheat JM, Raza $\mathrm{H}$, Kiat $\mathrm{H}$. The incremental value of SPECT/CT in characterizing solitary spine lesions. J Nucl Med Technol 2011;39(3):201-7

27. Sharma P, Jain TK, Reddy RM, Faizi NA, Bal C, Malhotra A, Kumar R. Comparison of single photon emission computed tomographycomputed tomography, computed tomography, single photon emission computed tomography and planar scintigraphy for characterization of isolated skull lesions seen on bone scintigraphy in cancer patients. Indian J Nucl Med 2014;29(1):22-9.

28. Bhatt G, Bhatt A, Dragun AE, Li XF, Civelek AC. Bisphosphonaterelated osteonecrosis of the jaw mimicking bone metastasis. Case Rep Oncol Med 2014;2014:281812.

29. Haraldsen A, Bluhme $H$, Røhl L, Pedersen EM, Jensen AB, Hansen EB, Nellemann H, Rasmussen F, Morsing A. Single photon emission computed tomography (SPECT) and SPECT/low-dose computerized tomography did not increase sensitivity or specificity compared to planar bone scintigraphy for detection of bone metastases in advanced breast cancer. Clin Physiol Funct Imaging 2016;36(1):40-6.

30. Kim CK, Park KW. Characteristic appearance of facet osteoarthritis of the lower lumbar spine on planar bone scintigraphy with a high negative predictive value for metastasis. Clin Nucl Med 2008;33(4):251-4.

31. Zhang Y, Li B, Shi H, Cai L, Hou J. Vertebral Hemangiolymphangioma Mimics Bone Metastases on 99mTc-MDP SPECT/CT. Clin Nucl Med. 2016;41(1):76-8.

32. Singh $H$, Sharma $P$, Naswa N, Nazar AH, Khangembam BC, Sahoo MK, Kumar R. Schmorl's node mimicking metastasis in a patient with breast cancer: diagnosis with $99 \mathrm{mTC}$ methylene diphosphonate SPECT-CT. Clin Nucl Med 2012;37(7):674-5.

33. Scheyerer MJ(1), Hüllner M, Pietsch C, Werner CM, Veit-Haibach $P$. Evaluation of pelvic ring injuries using SPECT/CT. Skeletal Radiol 2015;44(2):217-22.

34. Cook GJ, Azad GK, Goh V. Imaging Bone Metastases in Breast Cancer: Staging and Response Assessment. J Nucl Med 2016;57 Suppl 1:27S-33S.

35. Jambor I, Kuisma A, Ramadan S, Huovinen R, Sandell M, Kajander S, Kemppainen J, Kauppila E, Auren J, Merisaari H, Saunavaara J, Noponen T, Minn H, Aronen HJ, Seppänen M. Prospective evaluation of planar bone scintigraphy, SPECT, SPECT/CT, (18)F-NaF PET/ CT and whole body 1.5T MRI, including DWI, for the detection of bone metastases in high risk breast and prostate cancer patients: SKELETA clinical trial. Acta Oncol 2016;55(1):59-67.

36. Caglar M, Kupik O, Karabulut E, Høilund-Carlsen PF. Detection of bone metastases in breast cancer patients in the PET/CT era: Do we still need the bone scan? Rev Esp Med Nucl Imagen Mol 2016;35(1):3-11.

37. Hahn S, Heusner T, Kümmel S, Köninger A, Nagarajah J, Müller S, Boy C, Forsting M, Bockisch A, Antoch G, Stahl A. Comparison of FDG$\mathrm{PET} / \mathrm{CT}$ and bone scintigraphy for detection of bone metastases in breast cancer. Acta Radiol 2011;52(9):1009-14.

38. Chang CY, Gill CM, Joseph Simeone F, Taneja AK, Huang AJ, Torriani $\mathrm{M}$, Bredella MA. Comparison of the diagnostic accuracy of $99 \mathrm{~m}-\mathrm{Tc}$ MDP bone scintigraphy and 18F-FDG PET/CT for the detection of skeletal metastases. Acta Radiol 2016;57(1):58-65.

39. Harisankar CN, Agrawal K, Bhattacharya A, Mittal BR. F-18 fluorodeoxy-glucose and F-18 sodium fluoride cocktail PET/CT scan in patients with breast cancer having equivocal bone SPECT/CT. Indian J Nucl Med 2014;29(2):81-6.

40. Minamimoto $R$, Loening A, Jamali M, Barkhodari A, Mosci C, Jackson T, Obara P, Taviani V, Gambhir SS, Vasanawala S, lagaru A. Prospective Comparison of 99mTc-MDP Scintigraphy, Combined 18F-NaF and 18F-FDG PET/CT, and Whole-Body MRI in Patients with Breast and Prostate Cancer. J Nucl Med 2015;56(12):1862-8.

41. Greene LR, Wilkinson D. The role of general nuclear medicine in breast cancer. J Med Radiat Sci 2015;62(1):54-65.

42. Wuest W, Lell M, May MS, Saake M, Kuwert T, Uder M, Linke R. Thoracic non-osseous lesions in cancer patients detected in lowdose CT images acquired as part of skeletal SPECT/CT examinations. Nuklearmedizin 2015;54(4):173-7. 
43. Aras M, Erdil TY, Ones T, Dede F, Turoglu HT. Breast cancer lung metastases incidentally detected on bone SPECT/CT: a rare finding that might be missed on whole body scan. Rev Esp Med Nuc Imagen Mol 2014;33(3):191-2.

44. Uren RF, Howman-Giles R, Chung DK, Spillane AJ, Noushi F, Gillett D, Gluch L, Mak C, West R, Briody J, Carmalt H. SPECT/CT scans allow precise anatomical location of sentinel lymph nodes in breast cancer and redefine lymphatic drainage from the breast to the axilla. Breast 2012;21(4):480-6.

45. Serrano-Vicente J, Rayo-Madrid Jl, Domínguez-Grande ML, InfanteTorre JR,García-Bernardo L, Moreno-Caballero M, Medina-Romero F, Durán-Barquero C. Role of SPECT-CT in breast cancer sentinel node biopsy when internal mammary chain drainage is observed. Clin Transl Oncol 2016;18(4):418-25. [Epub ahead of print] doi: 10.1007/ s12094-015-1384

46. Lerman H, Metser U, Lievshitz G, Sperber F, Shneebaum S, Even-Sapir E. Lymphoscintigraphic sentinel node identification in patients with breast cancer: the role of SPECT-CT. Eur J Nucl Med Mol Imaging 2006;33(3):329-37.

47. Wagner T, Buscombe J, Gnanasegaran G, Navalkissoor S. SPECT/CT in sentinel node imaging. Nucl Med Commun 2013;34(3):191-202.

48. Jimenez-Heffernan A, Ellmann A, Sado H, Huić D, Bal C, Parameswaran R, Giammarile F, Pruzzo R, Kostadinova I, Vorster M, Almeida P, Santiago J, Gambhir S, Sergieva S, Calderon A, Young GO, ValdesOlmos R, Zaknun J, Magboo VP, Pascual TN. Results of a Prospective Multicenter International Atomic Energy Agency Sentinel Node Trial on the Value of SPECT/CT Over Planar Imaging in Various Malignancies. J Nucl Med 2015;56(9):1338-44.

49. Pouw B, Hellingman D, Kieft M, Vogel WV, van Os KJ, Rutgers EJ, Valdés Olmos RA, Stokkel MP. The hidden sentinel node in breast cancer: Reevaluating the role of SPECT/CT and tracer reinjection. Eur J Surg Oncol 2016;42(4):497-503. doi: 10.1016/j.ejso.2015.12.009.

50. Brouwer OR, Vermeeren $L$, van der Ploeg IM, Valdés Olmos RA, Loo CE, Pereira-Bouda LM, Smit F, Neijenhuis P, Vrouenraets BC, SivroPrndelj F, Jap-a-Joe SM, Borgstein PJ, Rutgers EJ, Oldenburg HS Lymphoscintigraphy and SPECT/CT in multicentric and multifocal breast cancer: does each tumour have a separate drainage pattern? Results of a Dutch multicentre study (MULTISENT). Eur J Nucl Med Mol Imaging 2012;39(7):1137-43.

51. Tan H, Yang B, Wu J, Wana S, Gu Y, Li W, Jiang Z, Qian M, Peng W. Localization and evaluation of sentinel lymph node in breast cancer from computed tomographic lymphography. J Comput Assist Tomogr 2011;35(3):367-74.

52. Na CJ, Kim J, Choi S, Han YH, Jeong HJ, Sohn MH, Youn HJ,Lim ST. The clinical value of hybrid sentinel lymphoscintigraphy to predict metastatic sentinel lymph nodes in breast cancer. Nucl Med Mol Imaging 2015;49(1):26-32.

53. Manca G, Volterrani D, Mazzarri S, Duce V, Svirydenka A, Giuliano A Mariani G. Sentinel lymph node mapping in breast cancer: a critical reappraisal of the internal mammary chain issue. Q J Nucl Med Mol Imaging 2014;58(2):114-26.

54. Thang SP, Tong AK, Ng DC. Postmastectomy/Axillary Node Dissection Chyloma: The Additional Value of SPECT/CT Lymphoscintigraphy. J Breast Cancer 2014;17(3):291-4.

55. Wadasaki K, Nishibuchi I. Relationship between sentinel lymph nodes and postoperative tangential fields in early breast cancer, evaluated using SPECT/CT. J Radiat Res 2015;56(5):835-40.

56. Reznik J, Cicchetti MG, Degaspe B, Fitzgerald TJ. Analysis of axillary coverage during tangential radiation therapy to the breast. Int J Radiat Oncol Biol Phys 2005;61(1):163-8.

57. Law M, Ma WH, Leung R, Li S, Wong KK, Ho WY, Kwong A. Evaluation of patient effective dose from sentinel lymph node lymphoscintigraphy in breast cancer: a phantom study with SPECT/CT and ICRP-103 recommendations. Eur J Radiol 2012;81(5):e717-20.

58. Van der Ploeg IMC, Valdés Olmos RA, Kroon BBR, et al. The Hybrid SPECT/CT as an Additional Lymphatic Mapping Tool in Patients with Breast Cancer. World Journal of Surgery 2008;32(9):1930-4.

59. Van der Ploeg IM, Nieweg OE, Kroon BB, et al. The yield of SPECT/CT for anatomical lymphatic mapping in patients with breast cancer. Eur J Nucl Med Mol Imaging 2009;36(6):903-9.

60. Moncayo VM, Aarsvold JN, Grant SF, Bartley SC, Alazraki NP. Status of sentinel lymph node for breast cancer. Semin Nucl Med 2013;43(4):281-93.

61. Shima H, Kutomi G, Satomi F, Maeda H, Takamaru T, Kameshima H, Omura T), Mori M, Hatakenaka M, Hasegawa T, Hirata K. Risk of node metastasis of sentinel lymph nodes detected in level II/III of the axilla by single-photon emission computed tomography/computed tomography. Exp Ther Med 2014;8(5):1447-52.

62. Vercellino L, Ohnona J, Groheux D, Slama A, Colletti PM, Chondrogiannis S, Merlet P, Toubert ME, Rubello D. Role of SPECT/ $\mathrm{CT}$ in sentinel lymph node detection in patients with breast cancer. Clin Nucl Med 2014;39(5):431-6.

63. Avramova-Cholakova S, Dimcheva M, Petrova E, Garcheva M Dimitrova M, Palashev $Y$, Vassileva J. Patient doses from hybrid SPECT-CT procedures. J.Radiat Prot Dosimetry 2015;165(1-4):424-9.

64. Sibille L, Chambert B, Alonso S, Barrau C, D'Estanque E, Al Tabaa Y, Collombier L, Demattei C, Kotzki PO, Boudousq V. Impact of the Adaptive Statistical iterative Reconstruction (ASiR) technique on radiation dose and image quality in bone SPECT/CT. Nucl Med. 2016. [Epub ahead of print] pii: jnumed.115.164772. 